\title{
Integrating Intelligent Electric Devices into Distributed Energy Resources in a Cloud- Based Environment
}

Petersen, Bo Søborg; Winther, D.; Pedersen, Anders Bro; Poulsen, Bjarne; Træholt, Chresten

Published in:

Proceedings of 2013 IEEE PES Innovative Smart Grid Technologies

Link to article, DOI:

10.1109/ISGTEurope.2013.6695276

Publication date:

2013

Link back to DTU Orbit

Citation (APA):

Petersen, B. S., Winther, D., Pedersen, A. B., Poulsen, B., \& Træholt, C. (2013). Integrating Intelligent Electric Devices into Distributed Energy Resources in a Cloud-Based Environment. In Proceedings of 2013 IEEE PES Innovative Smart Grid Technologies IEEE. https://doi.org/10.1109/ISGTEurope.2013.6695276

\section{General rights}

Copyright and moral rights for the publications made accessible in the public portal are retained by the authors and/or other copyright owners and it is a condition of accessing publications that users recognise and abide by the legal requirements associated with these rights.

- Users may download and print one copy of any publication from the public portal for the purpose of private study or research.

- You may not further distribute the material or use it for any profit-making activity or commercial gain

- You may freely distribute the URL identifying the publication in the public portal 


\title{
Integrating Intelligent Electric Devices into Distributed Energy Resources in a Cloud-Based Environment
}

\author{
B. Petersen, DTU Informatics, D. Winther, DTU Informatics, A. Pedersen, DTU Electrical \\ Engineering, B. Poulsen, DTU Informatics and C. Træholt, DTU Electrical Engineering
}

\begin{abstract}
Until now the main purpose of Distributed Energy Resources (DERs) has been to compliment the power plants. However, if DERs are to play a larger role in the power grid of the future, then improved communication and cooperation between these resources and the system operators is necessary. Cooperation requires intelligence at the level of the DER as well as at the aggregator level, and in order to efficiently facilitate this, communication must be easily achievable.

This project presents a proof-of-concept plug-and-play cloud solution for next generation DERs, built upon the IEC 61850[15] standard, that enable easy communication and cooperation between DERs and system operators.
\end{abstract}

Index Terms-Cloud, DER, Generic framework, Grid friendliness, IEC 61850, IED, Plug-and-play, Smart grid, WebSockets.

\section{INTRODUCTION}

A shift from conventional fossil-fuelled power plants to renewable energy sources is a major topic in today's discussions about the future of energy supply[5][6]. A lot of focus is aimed at replacing the power plants with DERs, which are smaller energy generation or consumption devices offering less than a few hundred $\mathrm{kW}$ of power, distributed across the power grid. Examples of such devices are solar panels, wind turbines, combined heat and power units and electric vehicles.

The transition from centralized power plants offering hundreds of MW to a grid of more distributed DERs introduce new challenges due to the power plants being the dominant factor in keeping the power grid stable. In order for the system operators to be able to manage the stability of the grid, the DERs must communicate and cooperate with each other and the system operators.

To achieve this, a solution, which enables central configuration and administration of DERs while facilitating distributed communication, needs to be developed. Furthermore, intelligent operation of the DERs should be achieved in order for them to cooperate [3].

Currently DERs produce energy and deliver it to the grid with little or no consideration to its state. Intelligent Electric Devices (IEDs), as oppose to DERs, operate as part of a network and can, due to their ability to process data, be used to control automated devices in a distribution grid. A combination of these two types of devices would result in a controllable and configurable intelligent distributed energy device (see Fig. 1), which would be ideal as a future grid friendly device assisting in maintaining the stability of the power grid.

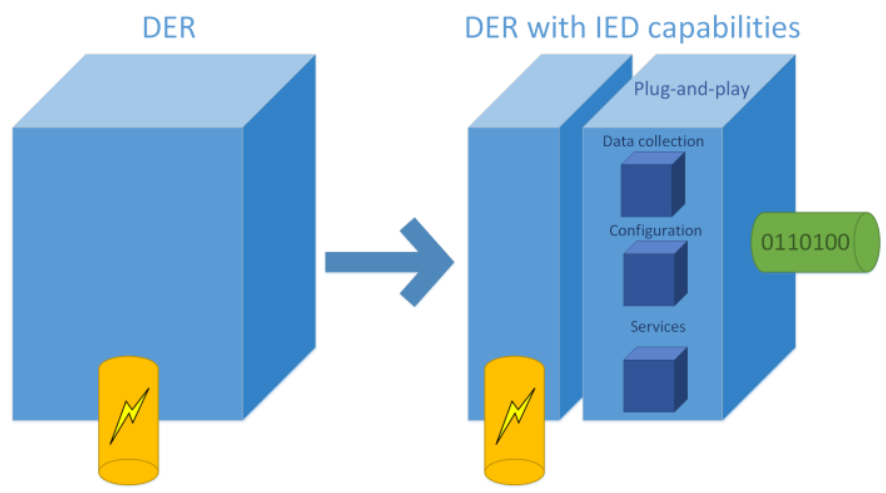

Fig. 1. DER upgraded to also become an IED, capable of participating in plug-and-play data collection, configuration and grid friendly control through the cloud.

Currently, the state of DERs is that they might selfishly on behalf of their owners or vendors. As an example, a future DER might decrease or entirely shut down the production of electricity, if the price of energy drops, to increase the profit of the owner [13]. This means, that the stability of the power grid will suffer under the independent administration of each DER.

Should the amount of such DERs be increased, the state of the power grid in question is very likely to destabilize due to the loss of control by the system operators. Therefore, to increase the integration of renewable energy a new kind of manageable DER that, among others, responds to requests for changes in production or consumption is needed.

In order for DERs to be centrally administrable, they must all be connected to a system, which facilitates monitoring and individual configuration of each distributed device. The simpler this connection and potential retrofitting of the units can be made; the easier it will be to achieve this goal. This asks for a plug-and-play data connection solution, which allows the devices to communicate with each other and a cloud-based service offering data collection, configuration and administration out of the box. 


\section{Cenelec Reference ARChitecture}

The main focus of this project is to develop a proof-ofconcept IEC 61850 compliant plug-and-play DER, acting as an IED, allowing it to connect to a cloud based data collection-, configuration- and administration system.

The device should be able to upload live data directly to the cloud, as well as to download new configurations, which can be changed by the parties who have been granted access. For the configuration of the devices to be fine-tuned in a meaningful way, data collected from the devices should be offered to stakeholders like manufacturers, owners and system administrators.

The CENELEC Smart Grid Reference Architecture [1] presents a description of the layers involved when building interoperable smart grid systems [8]. A high-level overview of these layers can be seen in Fig. 2.

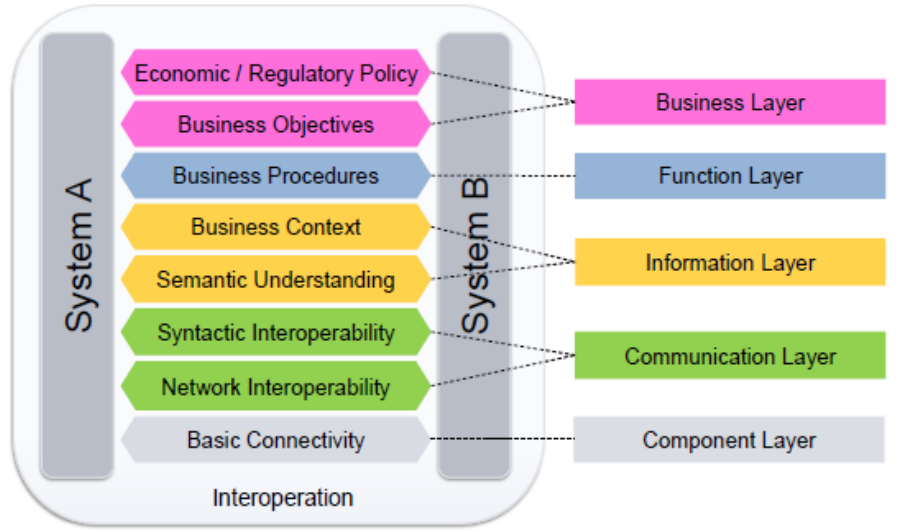

Fig. 2. Interoperability layers in the Smart Grid architecture model - [1] p. 26.

In general, these layers can be thought of as being dividable into either an Intelligent Electric Device Domain (IEDD) or an Application Domain (AD), with the Information Layer (IL) acting as a bridge transferring data between the domains.

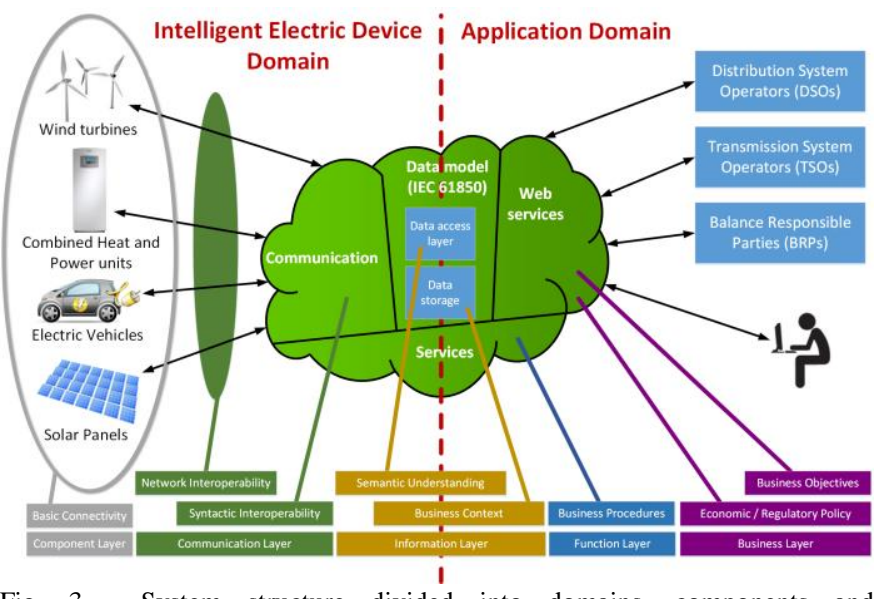

Fig. 3. System structure divided into domains, components and interoperability layers.

Fig. 3 present a mapping of these interoperability layers onto a cloud based data collection-, configuration- and administration system, featuring the components necessary in order to facilitate a generic and interoperable system for controlling DERs.

This architecture paves the way for stakeholders and services being able to monitor and make either transient or permanent changes to the configuration of every DER connected.

In order for the system to be able to communicate with each device, intelligence should be implemented, which help to maintain a constant connection to the cloud as well as to act as a generic interface capable of sending and receiving data. Upon connection, the device must identify itself by type, id and physical location ${ }^{1}$, as well as current configuration and capabilities. Based on these properties, services running in the cloud will be able to decide how to (re)configure the device as to make it prioritize the stability of the power grid. This behaviour is referred to as "plug-and-play for DERs".

\section{THE IEC 61850 STANDARD}

In order for the devices to be able to interoperate out of the box, a communication standard must be agreed upon and integrated into the DERs.

The IEC 61850 standard was originally developed for use within the area of power utility automation, but has later been expanded to incorporate a multitude of diverse scenarios. It features a communication model as well as a data model for communication among devices. Due to new extensions like the IEC 61850-7-420[22] and a constant development cycle to update the portfolio, the standard today covers most electrical devices and needs within the area.

This project mainly takes advantage of the data model described in IEC 61850-7[16][18]-[22] as well as the Substation Configuration Language (SCL) defined in IEC 61850-6[17].

As shown in Fig. 4, all data transmitted between the devices and the cloud is translated into SCL. This means that both the cloud as well as the DER units should implement the IEC 61850 data model in order to parse the data being sent. In practice, the DERs might only implement the subset of the standard that is necessary for them to operate, while the cloud must be able to parse the entire model as for it to work with all kinds of devices.

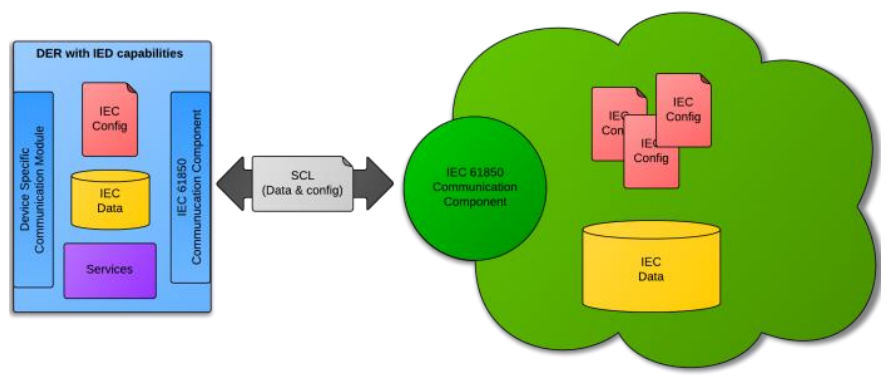

Fig. 4. DER to cloud communication using SCL as the data transfer medium.

Agreeing on a common communication language and data model as well as storing data on both the devices and the cloud eases the process of creating automated services for managing the vast amount of devices available. A service running in the cloud will only need to monitor device properties stored in the global IEC data model, as the devices

\footnotetext{
${ }^{1}$ Achievable by GPS or manual data entry.
} 
will take care of pushing live data at a fixed interval. In addition, the common interface of the DERs is a huge advantage when it comes to DER-to-DER communication and data sharing.

\section{Cloud ARChitecture}

In order for DERs to be able to communicate with a centralized system as well as other DERs, modern internet technologies are required, as the use of dedicated communication lines will be too expensive in this context. The communication should be initiated by the DERs connecting to an internet aggregator/control system. The cloud created fills the role of this internet aggregator/control system, by collecting data from the DERs as well as offering mechanisms for controlling them. The control can either be performed by services running in the cloud or in other control systems acting through web services offered by the cloud.

Fig. 3 shows how the cloud can work as an aggregator for Distribution System Operators (DSOs), Transmission System Operators (TSOs), Balance Responsible Parties (BRPs), Supervisory Control And Data Acquisition (SCADA) systems[10] and Virtual Power Plants (VPPs)[4], [7], [9] or that services can be run in the cloud to control the DERs.

The cloud contains an IEC 61850 data model, used both for persistent storage of data from the DERs and for actively accessing the data.

Persistent storage is implemented using a SQL database, with tables and attributes that match the IEC 61850 data model.

The data access layer is primarily composed of an ORM framework that maps the relational tables of the SQL database to the object oriented data model, implemented based on the IEC 61850 data model. On top of this runs RESTful Web services [11] through which the cloud-based control systems and system operators can access the data using XML[10]. These services can return raw data using the IEC 61850 path or accumulated/manipulated data, with the data communicated using SCL. Communication with the DERs also uses SCL, which maps the data directly to the data model used in the cloud.

With both the cloud and DERs using the IEC 61850 data model and SCL, no custom mapping is required; It is implicitly handled by the DER as part of its role as an IED.

Interoperable IEC 61850 based services can be run in the cloud, working directly with the data. This could be common services such as reporting and logging, or custom services specific to a group of DERs like power grid stabilization.

With common industry components being used like a SQL database and an ORM framework, the cloud can be run anywhere, only restricted by the programming language.

\section{DER ARCHITECTURE}

Three parts comprise the architecture of the improved DER unit (as shown in Fig 5):

- A device specific communication module.

- A conversion component.

- An IEC 61850 communication component.

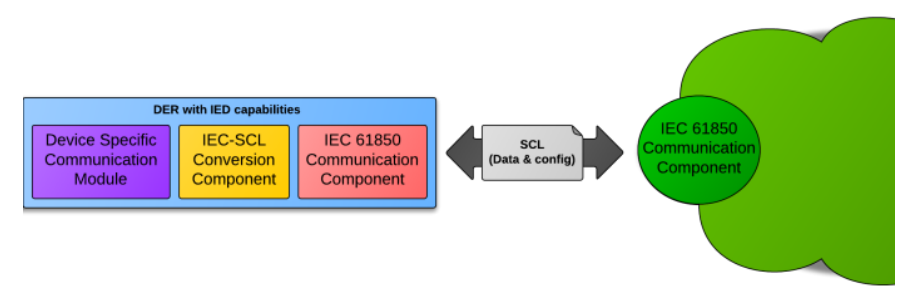

Fig. 5. DER architecture and communication.

The device specific communication module communicates with the hardware and uses the native interfaces of the DER to control and monitor settings and values. All monitored data is transformed into an IEC 61850 compatible data model and passed on to the conversion component.

The generic IEC-SCL conversion component is capable of parsing SCL formatted data in order to generate an IEC compliant logical device and vice versa. Thus, it functions as the middle layer between the device specific communication module and the IEC communication component. Whenever a logical device is received from the device specific communication module, it is transformed into SCL and passed on to the IEC 61850 communication component.

The generic IEC-SCL communication component is in charge of establishing and maintaining an open WebSocket connection to the cloud as well as to transfer SCL-formatted logical devices between the cloud and the IEC-SCL conversion component.

\section{CASE STUDIES}

In order to show and test the usefulness of the cloud based data collection-, configuration- and administration system, as well as the plug-and-play functionalities, a cloud system and a generic IEC 61850 based client for DERs has been implemented. The client works as a framework for implementing device specific modules for DERs, enabling them to send and receive IEC 61850 compliant data to the cloud service.

The case studies are centred around an Electric Vehicle Test bed (EVT)[2] which was developed to support development of electric vehicle control algorithms for the EDISON project[12]. Fig. 6 shows the setup featuring an array of batteries as well as a charger and an inverter, allowing the table to charge from- or send energy back into the grid. Internally it is controlled and monitored through various serial and TCP connections, which are connected to the T2G Module. This module is part of the IEC Client, which maps the entire table into a Logical Device and enables the table to connect seamlessly to the cloud service. 


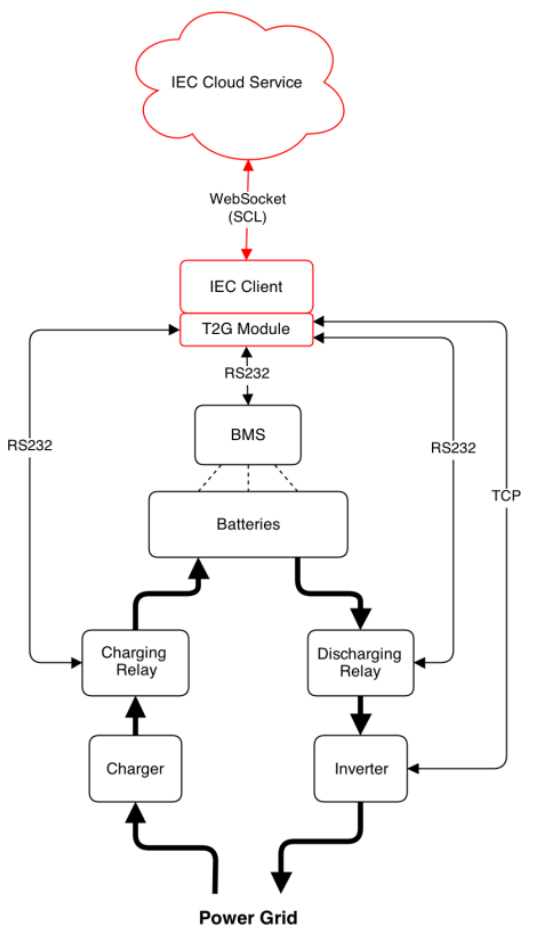

Fig. 6. EVT mapped to IEC 61850 and connected to cloud service.

The modules marked with red are the ones tested. The EVT could be any sort of DER offering data through one or several device specific communication interfaces. During tests the client software runs on a laptop connected to the interfaces of the table as well as the internet. The client is preconfigured with an IP address of the cloud service which runs on Microsoft Windows Azure[14].

\section{A. Case Study: Plug-and-play enabled DER}

Whenever the device is connected to the internet it automatically tries to initiate a connection to the cloud service. If it succeeds, a WebSocket connection is established between the two parties. The client then generates a logical device consisting of the logical node LPHD as well as other nodes relevant for that specific device. The logical nodes defining the EVT consist of ZBAT (Battery systems), ZBTC (Battery charger), DSCH (Energy schedule) and DSCC (Schedule control).

The LPHD node identifies the device by vendor, model, serial number and location, ZBAT and ZBTC holds the current values for voltage, current, capacity etc., while DSCH and DSCC shows which charging schedules are currently active on the EVT and how it can be updated. The logical device is translated into a SCL file and pushed to the cloud, which either creates a new device model (if the device has not been connected before) or adds the data to a model previously created.

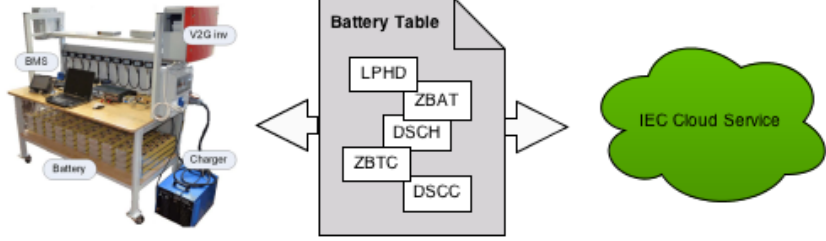

Fig. 7. Logical devices exchanged between cloud and Battery Table.

Having instantiated an open connection to the cloud, the client is free to update the logical devices and push new data to the cloud at any interval, thereby updating the global data model. Likewise, control properties and settings in the logical device might be changed in the cloud and pushed back to the DER, altering its behavior. For instance, when the EVT first identifies itself to the cloud, there might be a newer and better configuration available that forces all EVTs available to never discharge with more than a certain amperage. This configuration could be pushed automatically to the table, immediately after it has been connected.

\section{B. Case Study: Cloud monitoring and control of DER}

The EVT does not feature any protection against over or under charging of the batteries, which inspired the second case study. The system allows services to be hot-plugged into the cloud, allowing them to access and change properties and push the data back to the DER. In order to control the charging and discharging of the EVT a small service was implemented. When the EVT has connected to the cloud and starts to push live data, the State-of-Charge (SoC) is monitored by the service. This service is instructed to always keep the SoC of the EVT between $20 \%$ and $80 \%$.

When the EVT is connected to the cloud, as described in the first case study and the SoC drops below $20 \%$, the service will react by adding a new $0 \mathrm{~W}$ charging schedule to the DSCH node in the logical device. This schedule instructs the device to stop discharging the battery immediately. This charging schedule is pushed to the EVT together with a command telling the table to activate this schedule. The same happens if the SoC exceeds $80 \%$.

This service, which acts as a proof-of-concept, is mostly about protecting the EVT and it may not have much influence on the general stability of the power grid. However, the service could be changed into monitoring properties like voltage or frequency taking actions to prevent undesirable behaviour.

The DER might also have small services running locally, like the $\mathrm{SoC}$ protection service, which is controlled by the properties set from the cloud. In this aforementioned case, this would be the preferred option, as the unit would still be running the stabilizing services even if the connection to the cloud was lost.

\section{CONCLUSION}

The aim of this paper was to present a proof-of-concept version of an augmented DER incorporating functionalities that allows it to be part of a generic cloud based data collection-, configuration- and administration system.

Case studies showed how centralized configuration combined with a plug-and-play behaviour can be used to 
control and monitor resources with very little user interaction. The ability to add either device or type specific configurations directly to the cloud, makes it possible for vendors to push optimized configurations to new and old devices simply and efficient.

The consistent use of the standardised data model defined in IEC 61850 as well as the use of SCL for communication facilitates the dataflow between all interoperability layers throughout the IED Domain and the Application Domain. It also enables further advances within the area of DER-to-DER communication, by standardising the format of the data and properties available.

A centralized control system plays very well with decentralized services running on the DERs, as the cloud can be used to change parameters and fine tune the behaviour of each resource.

\section{REFERENCES}

\section{Technical Reports:}

[1] CEN-CENELEC-ETSI Smart Grid Coordination Group. Smart Grid Reference Architecture.

[2] F. Marra, D. Sacchetti, A. B. Pedersen, P. B. Andersen, C. Træholt, E. Larsen. Implementation of an Electric Vehicle Test Bed Controlled by a Virtual Power Plant for Contributing to Regulating Power Reserves. 2012 IEEE Power \& Energy Society General Meeting.

[3] L. Orda, J. Bach. A Generic Framework for Communication of Distributed Energy Resources through a Cloud-Based Service. (Unpublished)

[4] A. B. Pedersen and E. B Hauksson, Enabling distributed energy resources in a virtual power plant using IEC 61850, Master thesis. Technical University of Denmark. 2010.

[5] H. Farhangi. The Path of the smart grid. Power and Energy Magazine, IEEE, vol. 8, no. 1, pp. 18-28. 2010

[6] J. Driesen and F. Katiraei. Design for distributed energy resources. Power and Energy Magazine, IEEE, vol. 6, no. 3, pp. 30-40. 2008.

[7] S. You. Developing virtual power plant for optimized distributed energy resources operation and integration. Ph.D. dissertation. Ph.D. thesis. Technical University of Denmark. 2011.

[8] NIST. NIST Framework and Roadmap for Smart grid Interoperability standards.

http://www.nist.gov/public_affairs/releases/upload/smartgrid_interopera bility_final.pdf

[9] P. B. Andersen, B. Poulsen, C. Træholt, J. Østergaard. Using service oriented architecture in a generic virtual power plant. Information Technology: New Generations. 2009.

[10] S. A. Boyer. SCADA: Supervisory Control and Data Acquisition, ed.4. International Society of Automation. 2009.

[11] A. B. Pedersen, E. B. Hauksson, P. B. Andersen, B. Poulsen. C. Træholt, D. Gantenbein. Facilitating a generic communication interface to distributed energy ressources: Mapping IEC 61850 to RESTful services. Smart Grid Communications, IEEE, 2010.

[12] EDISON EV project (finished), http://www.edison-net.dk/default.aspx

[13] A prototype for European Smart Grids, EcoGrid EU http://www.eu-ecogrid.net/

[14] Microsoft Windows Azure, http://www.windowsazure.com

Standards:

[15] IEC 61850. Overview of 61850 and benefits. http://ieeexplore.ieee.org/xpl/login.jsp?tp=\&arnumber=1709546\&url=ht tp\%3A\%2F\%2Fieeexplore.ieee.org\%2Fxpls\%2Fabs_all.jsp\%3Farnumb er\%3D1709546

[16] IEC 61850-3:2012 ed.2 draft. General requirements.

[17] IEC 61850-6:2004 ed.1. Configuration description language for communication in electrical substations related to IEDs. http://webstore.iec.ch/webstore/webstore.nsf/Artnum_PK/43632

[18] IEC 61850-7-1:2011 ed.2. Principles and models. http://webstore.iec.ch/webstore/webstore.nsf/Artnum_PK/45336

[19] IEC 61850-7-2:2010 ed.2. Abstract communication service interface. http://webstore.iec.ch/webstore/webstore.nsf/Artnum_PK/44483
[20] IEC 61850-7-3:2011 ed.2. Common Data Classes. http://webstore.iec.ch/webstore/webstore.nsf/Artnum_PK/44733

[21] IEC 61850-7-4:2010 ed.2. Compatible logical node classes and data classes. http://webstore.iec.ch/webstore/webstore.nsf/Artnum_PK/43909

[22] IEC 61850-7-420: 2009 ed.1. Basic communication structure Distributed energy resources logical nodes. http://webstore.iec.ch/webstore/webstore.nsf/Artnum_PK/42706 